\title{
Reviewer Acknowledgements for Global Journal of Health Science, Vol. 7, No. 2
}

Global Journal of Health Science wishes to acknowledge the following individuals for their assistance with peer review of manuscripts for this issue. Their help and contributions in maintaining the quality of the journal is greatly appreciated.

Global Journal of Health Science is recruiting reviewers for the journal. If you are interested in becoming a reviewer, we welcome you to join us. Please find the application form and details at http://www.ccsenet.org/reviewer and e-mail the completed application form to gjhs@ccsenet.org.

\section{Reviewers for Volume 7, Number 2}

\begin{tabular}{|c|c|}
\hline Abolfazle Rahimi & Nazisa Hejazi \\
\hline Ama Pokuaa Fenny & $\mathrm{Na} Z \mathrm{hu}$ \\
\hline Amir Sayem & Pavlos Sarafis \\
\hline Ammar Eltayeb Ali Hassan & Polly Yeung \\
\hline Anjan Ghosh & Pelechas Eleftherios \\
\hline Brian Cook & Raywat Deonandan \\
\hline Emad Shdaifat & Robert Sloan \\
\hline Evangelia Mavrikaki & Samendra Sherchan \\
\hline Francisco Rodenas Rigla & Samira Schultz Mansur \\
\hline Gabriele Messina & Sara Melo \\
\hline Hadii Mamudu & Shilpee Singh \\
\hline José Mira & Sreenivas Phani Veeranki \\
\hline Kartheek R Balapala & Steven Hoffman \\
\hline Kimberley Geissler & Tarek Tawfik Amin \\
\hline Le Thi Thanh Xuan & Tugrul Erbaydar \\
\hline Loray Daws & Trisha Dunning \\
\hline Malliarou Maria & Wenhua Lu \\
\hline Mark Tully & Yasam Kemal Akpak \\
\hline Maya Hammoud & Zada Pajalic \\
\hline Meng Zhao & Zahra Fazli-Khalaf \\
\hline Montarat Thavorncharoensap & Zuhair Mohammedsaleh \\
\hline
\end{tabular}

Myo Nyein Aung 